\title{
SYNTHESIS AND ANTIMICROBIAL EVALUATION OF QUINAZOLINONE PEPTIDE DERIVATIVES
}

\author{
BHUPINDER KAPOOR ${ }^{1}$, ARSHID NABI ${ }^{2}$, REENA GUPTA $^{1}$, MUKTA GUPTA $^{1 *}$
}

${ }^{1}$ Department of Pharmaceutical Chemistry, School of Pharmaceutical Sciences, Lovely Professional University, Phagwara - 144 411, Punjab, India. ${ }^{2}$ Department of Chemistry, University of Malaya, Kuala - 50603, Lumpur, Malaysia. Email: mukta.16541@lpu.co.in

Received: 14 July 2017, Revised and Accepted: 25 July 2017

\section{ABSTRACT}

Objective: The increased microbial resistance against commercially available drugs initiated the development of novel and safe antimicrobial agents in last few decades. In this view, a series of amino acid/dipeptide derivatives of quinazolin-3(4H)-one was synthesized and was evaluated for their antimicrobial potential.

Method: Synthesis of amino acid/peptide derivatives were carried out by coupling 5-(2-(2-chlorophenyl)-4-oxoquinazolin-3(4H)-yl)-2-hydroxy benzoic acid with amino acid/dipeptide methyl esters in the presence of dicyclohexylcarbodiimide and $N$-methylmorpholine. The chemical structures of synthesized compounds were characterized by ${ }^{1} \mathrm{H}$ nuclear magnetic resonance and infrared spectroscopy and were screened for antibacterial activity by disc diffusion method.

Results: All the synthesized derivatives exhibited moderate to significant antibacterial activity against both Gram-positive and Gram-negative bacteria. The potency of compound $5 \mathrm{~d}$ was comparable to standard drug ciprofloxacin in all the strains of bacteria used. The compound $5 \mathrm{a}$ was found to be more active against Streptococcus pyogenes and Staphylococcus aureus while compound 5c against Pseudomonas aeruginosa and Escherichia coli.

Conclusion: Peptide derivatives of quinazolinone are promising antimicrobial agent and can be used for the synthesis of other novel compounds.

Keywords: Quinazolinone, Peptide, Antimicrobial agents.

(C) 2017 The Authors. Published by Innovare Academic Sciences Pvt Ltd.This is an open access article under the CC BY license (http://creativecommons. org/licenses/by/4. 0/) DOI: http://dx.doi.org/10.22159/ajpcr.2017.v10s4.21329

\section{INTRODUCTION}

Infectious diseases have become a major cause of motility worldwide, especially in immunocompromised patients such as patients suffering from tuberculosis, acquired immune deficiency syndrome, and cancer [1,2]. Although a number of antibiotics are commercially available for various infections but the development of microbial resistance is another challenge which is due to overuse of antibiotics and poor infection control practices [3-6].

Heterocyclic systems comprising quinazolinones have been explored to a major extent in last few decades due to its chemotherapeutic and antimicrobial potential. Quinazolinone (1) is a bicyclic compound containing benzene ring fused with pyrimidine ring. This heterocyclic molecule occupies a distinct place in the field of medicinal chemistry due to its wide spectrum of biological activities such as analgesic [7-11], antioxidant $[12,13]$, antimicrobial $[14,15]$, anticancer [16,17], antiinflammatory [18,19], antitubercular [20], antihyperlipidemic [21], antihistamine [22], antiviral [23], anticonvulsant [24], and antiparkinsonian [25].

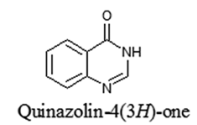

Peptides are heteropolymers and function as hormones, neurotransmitter, enzymes, substrate, and immunomodulators; hence considered as an important molecule in the field of health science [26-29]. Thus keeping in mind the therapeutic potential of quinazolinone as well as biodegradability and bioavailability profile of amino acids/peptide, an attempt has been made to synthesize amino acid/peptide derivatives of quinazolinone so as to increase its therapeutic potential and minimize adverse effects [30-33].

\section{METHODS}

\section{Chemistry}

All the chemicals such as tert-butoxycarbonyl protected amino acids (valine, tryptophan), amino acids (L-phenylalanine, L-leucine, L-serine, and L-arginine), dicyclohexylcarbodiimide (DCC), anthranilic acid, benzoyl chloride, $\mathrm{N}$-methylmorpholine (NMM), and trifluoroacetic acid (TFA) were purchased from Spectrochem Pvt. Ltd., (Mumbai, India). All the solvents were of commercial grade and distilled before use. Melting point of synthesized derivatives was determined by an open capillary method using digital melting point apparatus (Popular, India). The progress of reactions was monitored by thin layer chromatography (TLC) on silica gel $\mathrm{F}_{254}$ plates with visualization by ultraviolet or iodine vapors. The molecular structures of all the synthesized derivatives were confirmed by elemental analysis, infrared (IR), and ${ }^{1} \mathrm{H}$ nuclear magnetic resonance (NMR). The IR spectra (in KBr pellet) were recorded on FTIR-8400S (Shimadzu) spectrophotometer. Bruker Avance II ( $400 \mathrm{MHz}$ ) spectrometer was used to record ${ }^{1} \mathrm{H}$ NMR, and chemical shifts were given in $\delta$ (ppm) scale.

General scheme for synthesis of quinazolinone-amino acid/ peptide derivatives

The general scheme for the synthesis of quinazolinone nucleus and its amino acid/peptide derivatives has been provided in Figs. 1 and 2 .

\section{Experimental}

Synthesis of quinazolinone nucleus: (5-(2-(2-Chlorophenyl)-4oxoquinazolin-3-(4H)-yl)-2-hydroxy benzoic acid (5)

Synthesis of 2-(2-Chlorobenzamido) benzoic acid (3)

To a stirred solution of anthranilic acid $(10 \mathrm{mmol})$ in pyridine $(12 \mathrm{ml})$, 2 -chlorobenzoyl chloride ( $10 \mathrm{mmol}$ ) was added dropwise. The mixture obtained was further stirred at room temperature for $5 \mathrm{hrs}$. The resulting reaction mixture was filtered, washed with water and dried; followed by crystallization with ethanol to get pure compound. 


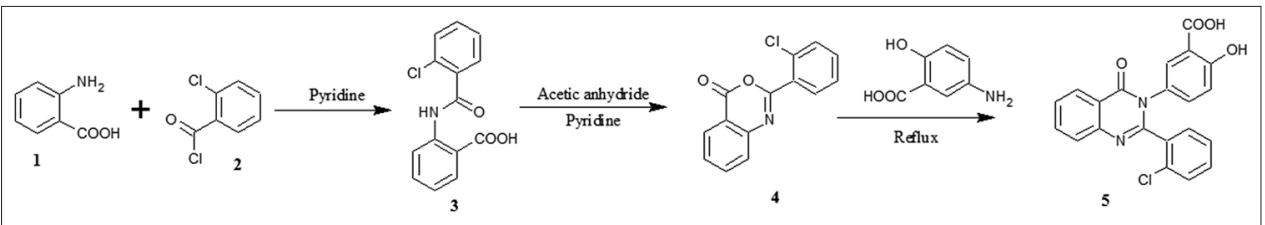

Fig. 1: Synthesis of quinazolinone nucleus

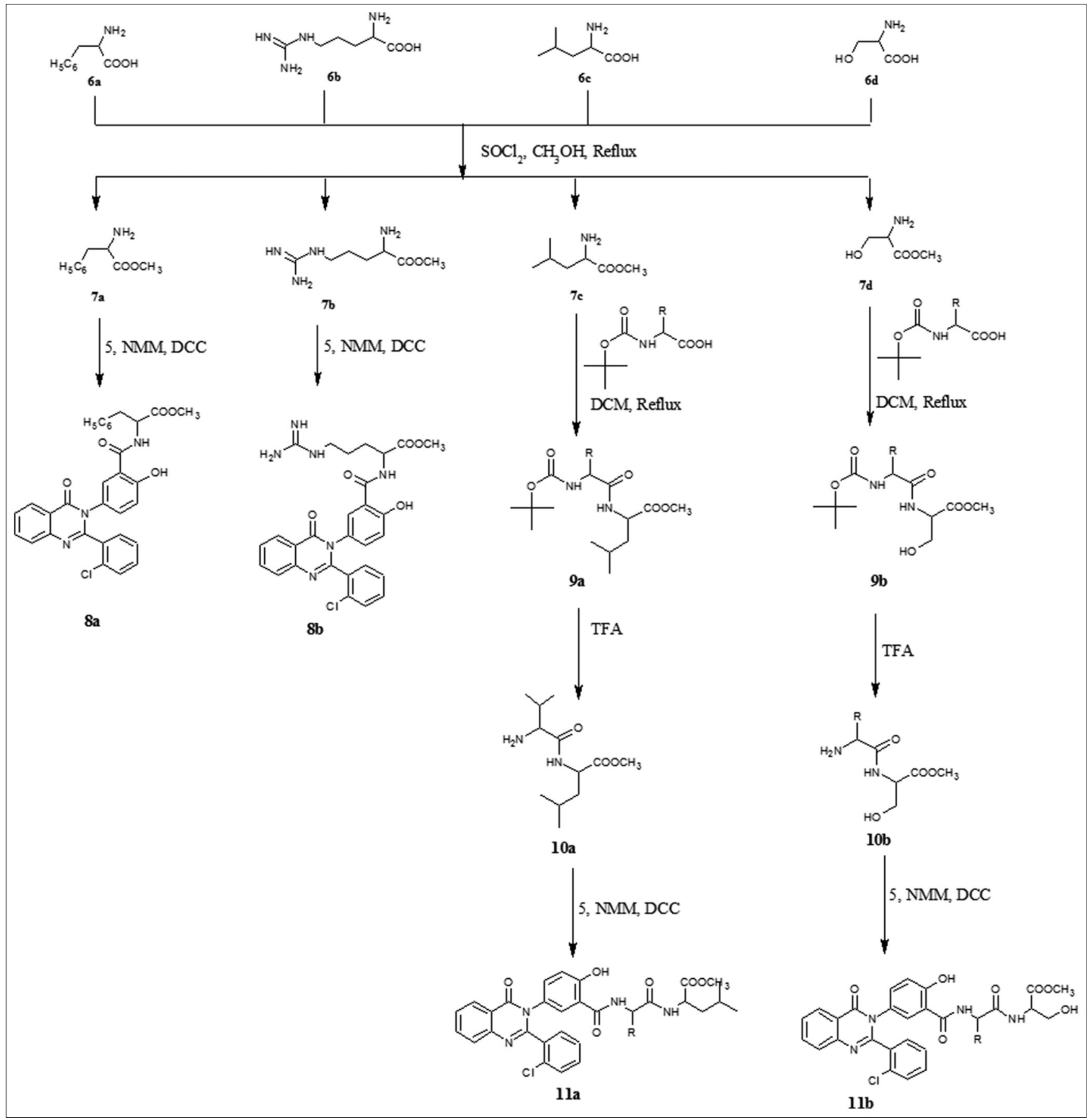

Fig. 2: Coupling of Boc-amino acid/peptides with quinazolinone nucleus

Synthesis of 2-(2-Chlorophenyl)-4H-benzo[d][1,3]oxazin-4-one (4) The solution of compound $3(20 \mathrm{mmol})$ in acetic anhydride $(34 \mathrm{ml})$ was refluxed for $6 \mathrm{hrs}$ or till the completion of reaction. The solvent was removed under reduced pressure; the precipitate was washed with petroleum ether and purified by crystallization with ethanol.
Synthesis of 5-(2-(2-Chlorophenyl)-4-oxoquinazolin-3(4H)-yl)-2hydroxy benzoic acid (5)

Equimolar amount of compound $4(16.4 \mathrm{mmol})$ and 5-aminosalicylic acid was heated on a water bath at $200^{\circ} \mathrm{C}$ for $2 \mathrm{hrs}$. The separated solid was crystallized with ethanol to get pure compound. 
Preparation of L-amino acid methyl ester hydrochloride (7a-d) Esterification of amino acids $6 \mathrm{a}-\mathrm{d}(10 \mathrm{mmol})$ was carried out by conversion into acid chloride using thionyl chloride $(10 \mathrm{mmol})$, followed by addition of methanol at $0^{\circ} \mathrm{C}$. The reaction mixture was refluxed on a water bath for $12 \mathrm{hrs}$ at a temperature of $110^{\circ} \mathrm{C}$. The progress of the reaction was monitored by TLC. After the completion of the reaction, methanol was removed under reduced pressure and the treated with ether to remove dimethyl sulfite. The purification of the product was carried out by recrystallization with methanol $[34,35]$.

\section{Preparation of Boc-dipeptide methyl ester (9a-b)}

To the solution of amino acid methyl ester hydrochloride $7 \mathrm{c}$ and $7 \mathrm{~d}(20$ $\mathrm{mmol})$ in chloroform $(40 \mathrm{ml}), \mathrm{NMM}(42 \mathrm{mmol})$ was added at $0^{\circ} \mathrm{C}$, and the mixture was stirred for 15 minutes. Then, Boc-amino acid ( $20 \mathrm{mmol})$ in chloroform $(40 \mathrm{ml})$ and DCC $(20 \mathrm{mmol})$ was added while stirring the reaction mixture. After stirring for $48 \mathrm{hrs}$, the reaction mixture was filtered, and residue was washed with chloroform $(30 \mathrm{ml})$ and added to the filtrate. The combined filtrate was further extracted with $25 \mathrm{ml}$ of $5 \%$ sodium hydrogen carbonate and then with $25 \mathrm{ml}$ saturated sodium chloride solution. After drying of the organic layer, the solvent was removed under vacuum by rotary evaporator. The crude product obtained was purified by crystallization using a mixture of chloroform and petroleum ether $[26,36]$.

\section{Deprotection of dipeptide methyl ester at amino end (10a-b)}

Deprotection of Boc group was carried out by dissolving Boc-dipeptide methyl ester $(10 \mathrm{mmol})$ in chloroform $(15 \mathrm{ml})$ followed by addition of TFA $(20 \mathrm{mmol})$. The resulting solution was stirred for 30 minutes. Then, the reaction mixture was concentrated, dried and washed with a mixture of chloroform and petroleum ether [37-39].

Synthesis of amino acid/peptide derivatives of 5-(2-(2 Chlorophenyl)-4-oxoquinazolin-3(4H)-yl)-2-hydroxy benzoic acid (8a-b, 11a-b)

Amino acid methyl ester/deprotected dipeptide methyl ester (1 mmol) was dissolved in THF; NMM $(0.01 \mathrm{mmol})$ was added at $0^{\circ} \mathrm{C}$, and the reaction mixture was stirred for 15 minutes. In another beaker, a solution of compound $5(1 \mathrm{mmol})$ in THF $(7.4 \mathrm{ml})$ and DCC $(0.01 \mathrm{mmol})$ was prepared and added to reaction mixture with stirring. After stirring for $36 \mathrm{hrs}$, the reaction mixture was filtered, and the residue was washed with THF $(5 \mathrm{ml})$, followed by washing with $5 \%$ sodium hydrogen carbonate and saturated sodium chloride solutions $(15 \mathrm{ml})$. The organic layer was dried over anhydrous magnesium sulfate and evaporated by rotary evaporator. The purification of the crude product was carried out by recrystallization with a mixture of chloroform and n-hexane (Table 1).

\section{Spectral analysis of synthesized compounds}

5-(2-(2-Chlorophenyl)-4-oxoquinazolin-3(4H)-yl)-2-hydroxy benzoic acid (5)

FTIR ( $\mathrm{KBr}, \mathrm{cm}^{-1}$ ): 3356-3285 (O-H, str.), 3076 (C-H str. aromatic),1725 (C=0 str. acid), 1665 ( $\mathrm{C}=0$ str. amide), 775 ( $\mathrm{C}-\mathrm{Cl}$ str.), 750 (ortho substitution oop); ${ }^{1} \mathrm{H}$ NMR (400 MHz, DMSO, $\delta$ ppm): 12.1 (s, $\mathrm{COOH}, 1 \mathrm{H}$ ), 8.18 (s, Ar-H, 1H, benzoic acid), 8.13-7.64 (m, Ar- $\mathrm{H}, 4 \mathrm{H}$, quinazoline), 7.51-7.42 (m, Ar- $\mathrm{H}, 4 \mathrm{H}$, chlorobenzene), 7.39-7.36 (m, Ar-H, 2H, benzoic acid), $3.8(\mathrm{~s}, \mathrm{OH}, 1 \mathrm{H})$.

\section{L-Phenylalanine methyl ester hydrochloride (7a)}

FTIR (KBr, cm${ }^{-1}$ ): 3423 and 3368 (primary N-H str.), 2926 (C-H str. aromatic), 2852 (C-H str. aliphatic), 1745 (C=0 str. ester), 1238 (C-0 str.), 762 and 695 (mono substitution oop); ${ }^{1} \mathrm{H}$ NMR (400MHz, DMSO, $\delta$ ppm): $8.71\left(\mathrm{~d}, \mathrm{NH}_{2}, 2 \mathrm{H}\right), 7.19-714(\mathrm{~m}, \mathrm{Ar}-\mathrm{H}, 5 \mathrm{H}), 3.66\left(\mathrm{~s}, \mathrm{OCH}_{3}, 3 \mathrm{H}\right), 3.54$ (m, $\mathrm{CH}_{2}$ benzyl, 2H), $3.32(\mathrm{~m}, \mathrm{CH}, 1 \mathrm{H})$.

\section{L-Arginine methyl ester hydrochloride (7b)}

FTIR (KBr, cm${ }^{-1}$ ): 3456 and 3370 (primary N-H str), 2955 (C-H str. aliphatic), 1743(C=0, ester), 1200 (C-O str.); ${ }^{1} \mathrm{H}$ NMR (400MHz, DMSO, $\delta \mathrm{ppm}): 8.76\left(\mathrm{~d}, \mathrm{NH}_{2}, 2 \mathrm{H}\right), 7.84$ (s, terminal $\mathrm{NH}$ of guanidine group, $\left.1 \mathrm{H}\right)$, $6.63\left(\mathrm{~m}\right.$, terminal $\mathrm{NH}_{2}$ of guanidine group, $\left.2 \mathrm{H}\right), 3.69\left(\mathrm{~s}, \mathrm{OCH}_{3}, 3 \mathrm{H}\right), 3.36$ (m, $\left.\mathrm{CH}-\mathrm{NH}_{2}, 1 \mathrm{H}\right), 3.28(\mathrm{~m}, \mathrm{CH}-\mathrm{NH}, 2 \mathrm{H}), 1.8-1.6\left(\mathrm{~m}, \mathrm{CH}_{2}, 4 \mathrm{H}\right)$.

\section{L-Leucine methyl ester hydrochloride (7c)}

FTIR (KBr, cm${ }^{-1}$ ): 3462 and 3390 (primary N-H str), 2872 (C-H str. aliphatic), 1739 (C=0, ester) 1240 (C-0 str); ${ }^{1} \mathrm{H}$ NMR (400 MHz, DMSO, $\delta \mathrm{ppm}): 8.63\left(\mathrm{~d}, \mathrm{NH}_{2}, 2 \mathrm{H}\right), 3.61\left(\mathrm{~s}, \mathrm{OCH}_{3}, 3 \mathrm{H}\right), 3.36\left(\mathrm{~m}, \mathrm{CH}-\mathrm{NH}_{2}, 1 \mathrm{H}\right), 1.87$ $\left(\mathrm{m}, \mathrm{CH}_{2}, 2 \mathrm{H}\right), 1.49(\mathrm{~m}, \mathrm{CH}, 1 \mathrm{H}), 0.90-0.96\left(\mathrm{~m}, \mathrm{CH}_{3}, 6 \mathrm{H}\right)$.

\section{L-Serine methyl ester hydrochloride (7d)}

FTIR ( $\mathrm{KBr}, \mathrm{cm}^{-1}$ ): 3550 (O-H str.), 2996 (C-H str. aliphatic), $1760(\mathrm{C}=0$, ester), 1249 (C-O str.); ${ }^{1} \mathrm{H}$ NMR (400 MHz, DMSO, $\delta$ ppm): 8.96 (d, $\mathrm{NH}_{2}$, $2 \mathrm{H}), 4.94(\mathrm{t}, \mathrm{OH}, 1 \mathrm{H}), 4.26(\mathrm{t}, \mathrm{CH} 2,2 \mathrm{H}), 3.66\left(\mathrm{~s}, \mathrm{OCH}_{3}, 3 \mathrm{H}\right), 3.46(\mathrm{~m}, \mathrm{CH}-$ $\left.\mathrm{NH}_{2}, 1 \mathrm{H}\right)$.

\section{tert-Butyloxycarbonyl-valine-leucine methyl ester (9a)}

FTIR $\left(\mathrm{KBr}, \mathrm{cm}^{-1}\right.$ ): 3350 (secondary N-H str.), 2978 (C-H str. aliphatic), 1756 ( $\mathrm{C}=0$ str. ester), 1680 ( $\mathrm{C}=0$ str. amide), 1210 (C-O str.); ${ }^{1} \mathrm{H}$ NMR (400 MHz, DMSO, $\delta$ ppm): $8.32(\mathrm{~d}, \mathrm{NH}, 1 \mathrm{H}), 7.39(\mathrm{~d}, \mathrm{NH}, 1 \mathrm{H}), 4.51(\mathrm{~m}$, $\mathrm{CH}, 1 \mathrm{H}), 4.34(\mathrm{~m}, \mathrm{CH}, 1 \mathrm{H}), 3.68\left(\mathrm{~s}, \mathrm{OCH}_{3}, 3 \mathrm{H}\right), 2.73(\mathrm{~m}, \mathrm{CH}, 1 \mathrm{H}), 1.81(\mathrm{~m}$, $\left.\mathrm{CH}_{2}, 2 \mathrm{H}\right), 1.42(\mathrm{~m}, \mathrm{CH}, 10 \mathrm{H}), 0.96-0.90\left(\mathrm{~m}, \mathrm{CH}_{3}, 12 \mathrm{H}\right)$

\section{tert-Butyloxycarbonyl-tryptophenyl-serine methyl ester (9b)}

FTIR ( $\mathrm{KBr}, \mathrm{cm}^{-1}$ ): 3590 (O-H str.), 3235 (secondary N-H str.), 3090 (C-H str. aromatic), 2985 (C-H str. aliphatic), 1758 (C=0 str. ester), 1692 (C=0 str. amide), 1270 (C-O str.); ${ }^{1} \mathrm{H}$ NMR (400MHz, DMSO, $\delta$ ppm): 11.6 (d, $\mathrm{NH}$ of tryptophan ring, $1 \mathrm{H}), 8.3-8.1(\mathrm{~d}, \mathrm{NH}, 2 \mathrm{H}), 7.41-7.10(\mathrm{~m}, \mathrm{Ar}-\mathrm{H}$ tryptophan ring, $5 \mathrm{H}), 5.82(\mathrm{~d}, \mathrm{CH}, 1 \mathrm{H}), 4.94(\mathrm{t}, \mathrm{OH}, 1 \mathrm{H}), 4.26\left(\mathrm{t}, \mathrm{CH}_{2}, 2 \mathrm{H}\right)$, $4.02(\mathrm{~m}, \mathrm{CH}, 1 \mathrm{H}), 3.58\left(\mathrm{~s}, \mathrm{OCH}_{3}, 3 \mathrm{H}\right), 1.46\left(\mathrm{~m}, \mathrm{CH}_{3}, 9 \mathrm{H}\right)$.

\section{Valine-leucine methyl ester (10a)}

FTIR (KBr, cm $\left.{ }^{-1}\right): 3420$ \&3350 (primary N-H str.), 2954 (C-H str. aliphatic), 1762 ( $\mathrm{C}=0$ str. ester), 1674 ( $\mathrm{C}=0$ str. amide), 1219 (C-0 str.); ${ }^{1} \mathrm{H}$ NMR $(400 \mathrm{MHz}, \mathrm{DMSO}, \delta \mathrm{ppm}): 8.32(\mathrm{~d}, \mathrm{NH}, 1 \mathrm{H}), 6.5\left(\mathrm{~d}, \mathrm{NH}_{2}, 2 \mathrm{H}\right)$, $4.54(\mathrm{~m}, \mathrm{CH}, 1 \mathrm{H}), 3.61\left(\mathrm{~s}, \mathrm{OCH}_{3}, 3 \mathrm{H}\right), 3.37(\mathrm{~m}, \mathrm{CH}, 1 \mathrm{H}), 2.06(\mathrm{~m}, \mathrm{CH}, 1 \mathrm{H})$, $1.81\left(\mathrm{~m}, \mathrm{CH}_{2}, 2 \mathrm{H}\right), 1.42(\mathrm{~m}, \mathrm{CH}, 10 \mathrm{H}), 0.96-0.90\left(\mathrm{~m}, \mathrm{CH}_{3}, 12 \mathrm{H}\right)$.

\section{Tryptophan-serine methyl ester (10b)}

FTIR (KBr, cm ${ }^{-1}$ ): 3598 (O-H str), 3224 (secondary N-H str.), 3082 (C-H str. aromatic), 2973 (C-H str. aliphatic), 1767 (C=0 str. ester), 1696 ( $\mathrm{C}=0$ str. amide), 1296 (C-O str.); ${ }^{1} \mathrm{H}$ NMR (400MHz, DMSO, $\delta$ ppm): 11.24 (d, NH of tryptophan ring, $1 \mathrm{H}), 8.32(\mathrm{~d}, \mathrm{NH}, 1 \mathrm{H}), 7.46-7.19(\mathrm{~m}, \mathrm{Ar}-\mathrm{H}$ tryptophan ring, $5 \mathrm{H}), 6.7\left(\mathrm{~s}, \mathrm{NH}_{2}, 2 \mathrm{H}\right) 4.86(\mathrm{t}, \mathrm{OH}, 1 \mathrm{H}), 4.6(\mathrm{t}, \mathrm{CH}, 1 \mathrm{H})$, $4.26\left(\mathrm{~m}, \mathrm{CH}_{2}, 2 \mathrm{H}\right), 4.02(\mathrm{~m}, \mathrm{CH}, 1 \mathrm{H}), 3.63\left(\mathrm{~s}, \mathrm{OCH}_{3}, 3 \mathrm{H}\right)$.

5-(2-(2-Chlorophenyl)-4-oxoquinazolin-3(4H)-yl)-2hydroxybenzoyl-phenaylalanine methyl ester (8a)

FTIR (KBr, cm ${ }^{-1}$ ): 3486-3452(O-H str), 3054 (C-H str. aromatic), 2958 (C-H str. aliphatic), 1743 ( $\mathrm{C}=0$ str. ester), 1670 ( $\mathrm{C}=0$ str. amide), 1608 and 1458 (C=C str. aromatic), 1338 (C-0 str.), 1219 (C-N str.), 782(C-Cl str.); ${ }^{1} \mathrm{H}$ NMR (400 MHz, DMSO, $\delta$ ppm): 9.87 (d, NH, 1H, amide), 8.18 (s, Ar- $\mathrm{H}, 1 \mathrm{H}$, benzoic acid), 8.13-7.64 (m, Ar- $\mathrm{H}, 4 \mathrm{H}$, quinazoline), 7.51-7.42 (m, Ar- $\mathrm{H}, 4 \mathrm{H}$, chlorobenzene), 7.19-7.14 (m, Ar-H, 5H), 4.84 (d, CH, 1H), $3.96(\mathrm{~s}, \mathrm{OH}, 1 \mathrm{H}), 3.66\left(\mathrm{~s}, \mathrm{CH}_{3}, 3 \mathrm{H}\right), 3.27\left(\mathrm{~d}, \mathrm{CH}_{2}, 2 \mathrm{H}\right)$.

5-(2-(2-Chlorophenyl)-4-oxoquinazolin-3(4H)-yl)-2hydroxybenzoyl-arginine methyl ester $(8 b)$

FTIR (KBr, cm ${ }^{-1}$ ): 3469-3427 (O-H str.), 3244 and 3212 (N-H str.), 3076 (C-H str. aromatic), 2972 (C-H str. aliphatic), 1756 (C=0 str. ester), 1689 (C=0 str. amide), 1624 and 1470 (C=C str. aromatic), 1342 (C-0 str.), 1235 (C-N str.), 756 (C-Cl str.); ${ }^{1} \mathrm{H}$ NMR (400 MHz, DMSO, $\delta$ ppm): 8.87 (d, NH, 1H, amide), 8.20 (s, Ar-H, 1H, benzoic acid), 8.09-7.72 (m, Ar-H, $4 \mathrm{H}$, quinazoline), 7.81 (s, terminal $\mathrm{NH}$ of guanidine group, $1 \mathrm{H}$ ), 7.53-7.49 (m, Ar- $\mathrm{H}, 4 \mathrm{H}$, chlorobenzene), 6.52 (m, terminal $\mathrm{NH}_{2}$ of guanidine group, 
Table 1: Physicochemical properties of synthesized compounds

\begin{tabular}{|c|c|c|c|c|c|c|}
\hline Compounds & Molecular formula & Molecular weight & $\mathbf{R}$ & Yield (\%) & M.p $\left({ }^{\circ} \mathrm{C}\right)$ & $\mathbf{R}_{\text {fvalue }}{ }^{a}$ \\
\hline 5 & $\mathrm{C}_{12} \mathrm{H}_{13} \mathrm{ClN}_{2} \mathrm{O}_{4}$ & 392.79 & - & 71 & $127-130$ & 0.81 \\
\hline $7 a$ & $\mathrm{C}_{10} \mathrm{H}_{14} \mathrm{H}_{13} \mathrm{ClN}_{2}$ & 215.67 & - & 92 & $161-164$ & 0.79 \\
\hline $7 b$ & $\mathrm{C}_{7} \mathrm{H}_{17} \mathrm{ClN}_{4} \mathrm{O}_{2}$ & 224.68 & - & 88 & $155-162$ & 0.64 \\
\hline $7 \mathrm{c}$ & $\mathrm{C}_{7} \mathrm{H}_{16} \mathrm{ClNO}_{2}$ & 181.66 & - & 72 & $155-159$ & 0.62 \\
\hline $7 \mathrm{~d}$ & $\mathrm{C}_{4} \mathrm{H}_{10} \mathrm{ClNO}_{3}$ & 155.58 & - & & & \\
\hline $9 a$ & $\mathrm{C}_{17} \mathrm{H}_{32} \mathrm{~N}_{2} \mathrm{O}_{5}^{3}$ & 344.44 & $-\mathrm{CH}\left(\mathrm{CH}_{3}\right)_{2}$ & 70 & $143-145$ & 0.86 \\
\hline $9 b$ & $\mathrm{C}_{20} \mathrm{H}_{27} \mathrm{~N}_{3} \mathrm{O}_{6}$ & 405.44 & & 78 & $160-162$ & 0.91 \\
\hline $10 \mathrm{a}$ & $\mathrm{C}_{11} \mathrm{H}_{2} \mathrm{~N}_{2} \mathrm{O}_{3}$ & 244.33 & $-\mathrm{CH}\left(\mathrm{CH}_{z}\right.$ & 89 & $172-177$ & 0.85 \\
\hline $10 \mathrm{~b}$ & $\mathrm{C}_{15}^{12} \mathrm{H}_{19}^{24} \mathrm{~N}_{3} \mathrm{O}_{4}$ & 305.32 & & 66 & $169-171$ & 0.77 \\
\hline $8 a$ & $\mathrm{C}_{31} \mathrm{H}_{24} \mathrm{ClN}_{3} \mathrm{O}_{5}$ & 553.99 & - & 76 & $159-161$ & 0.76 \\
\hline $8 b$ & $\mathrm{C}_{28} \mathrm{H}_{27} \mathrm{ClN}_{6} \mathrm{O}_{5}$ & 563.00 & - & 69 & $145-151$ & 0.64 \\
\hline $11 \mathrm{a}$ & $\mathrm{C}_{33}^{28^{1} \mathrm{H}_{25} \mathrm{H}_{35} \mathrm{Cl}_{4} \mathrm{O}_{6}}$ & 619.10 & $-\mathrm{CH}\left(\mathrm{CH}_{3}\right.$ & 73 & $132-134$ & 0.67 \\
\hline $11 b$ & $\mathrm{C}_{36} \mathrm{H}_{30} \mathrm{ClN}_{5} \mathrm{O}_{7}$ & 680.10 & & 65 & $161-164$ & 0.71 \\
\hline
\end{tabular}

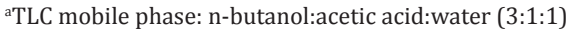

Table 2: Antimicrobial activity of quinazolinone peptide derivatives

S.No. Compounds Zone of inhibition (mm)

S. pyogenes S. aureus P. aeruginosa E. coli

\begin{tabular}{|c|c|c|c|c|c|}
\hline 1 & $8 a$ & $18 \pm 0.56$ & $19 \pm 0.13$ & $16 \pm 0.7$ & $14 \pm 0.45$ \\
\hline 2 & $8 b$ & $6 \pm 0.87$ & $13 \pm 0.3$ & $10 \pm 0.55$ & $11 \pm 0.7$ \\
\hline 3 & $11 \mathrm{a}$ & $15 \pm 0.9$ & $16 \pm 0.1$ & $19 \pm 0.2$ & $18 \pm 0.48$ \\
\hline 4 & $11 b$ & $19 \pm 0.2$ & $22 \pm 0.11$ & $20 \pm 0.32$ & $18 \pm 0.5$ \\
\hline 5 & Ciprofloxacin & $23 \pm 0.12$ & $25 \pm 0.19$ & $27 \pm 0.78$ & $22 \pm 0.43$ \\
\hline
\end{tabular}

Zone of inhibition is expressed as mean \pm standard deviation of triplicates.

S. pyogenes: Streptococcus pyogenes, S. aureus: Staphylococcus aureus,

Pseudomonas aeruginosa: P. aeruginosa, E. coli: Escherichia coli

$2 \mathrm{H}), 4.51(\mathrm{~d}, \mathrm{CH}, 1 \mathrm{H}), 4.02(\mathrm{~s}, \mathrm{OH}, 1 \mathrm{H}), 3.74\left(\mathrm{~s}, \mathrm{CH}_{3}, 3 \mathrm{H}\right), 3.34\left(\mathrm{~d}, \mathrm{CH}_{2}, 2 \mathrm{H}\right)$, $2.5(\mathrm{~m}, \mathrm{NH}$ of guanidine group, $1 \mathrm{H}), 1.92\left(\mathrm{~m}, \mathrm{CH}_{2}, 2 \mathrm{H}\right), 1.51\left(\mathrm{~m}, \mathrm{CH}_{2}, 2 \mathrm{H}\right)$.

5-(2-(2-Chlorophenyl)-4-oxoquinazolin-3(4H)-yl)-2hydroxybenzoyl-valine-leucine methyl ester (11a)

FTIR (KBr, cm ${ }^{-1}$ ): 3456-3399 (O-H str.), 3050 (C-H str. aromatic), 2947 (C-H str. aliphatic), 1765 (C=0 str. ester), 1660 (C=0 str. amide), 1635 and 1475 (C=C str. aromatic), 1290 (C-0 str.), 1260 (C-N str.), 742 (C-Cl str.); ${ }^{1} \mathrm{H}$ NMR (400 MHz, DMSO, $\delta$ ppm): 8.88 and 8.65 (d, NH, $2 \mathrm{H}$, amide), 8.24 (s, Ar- $\mathrm{H}, 1 \mathrm{H}$, aromatic), 8.13-7.68 (m, Ar- $\mathrm{H}, 4 \mathrm{H}$, quinazoline), 7.51$7.42(\mathrm{~m}, \mathrm{Ar}-\mathrm{H}, 4 \mathrm{H}$, chlorobenzene), $4.84(\mathrm{~d}, \mathrm{CH}, 1 \mathrm{H}), 4.51(\mathrm{~m}, \mathrm{CH}, 1 \mathrm{H})$, 4.24 (s, OH, 1H), $3.74\left(\mathrm{~s}, \mathrm{CH}_{3}, 3 \mathrm{H}\right), 1.87\left(\mathrm{~m}, \mathrm{CH}_{2}, 2 \mathrm{H}\right), 1.49(\mathrm{~m}, \mathrm{CH}, 1 \mathrm{H})$, 0.96-0.9 (m, $\left.\mathrm{CH}_{3}, 12 \mathrm{H}\right)$.

5-(2-(2-Chlorophenyl)-4-oxoquinazolin-3(4H)-yl)-2hydroxybenzoyl-tryptophan-serine methyl ester $(11 \mathrm{~b})$

FTIR (KBr, cm ${ }^{-1}$ ): 3468-3412 (O-H str.), 3069 (C-H str. aromatic), 2960 (C-H str. aliphatic), 1772(C=0 str. ester), 1675(C=0 str. amide), 1638 and 1482 (C=C str. aromatic), 1286(C-O str.), 1254(C-N str.), 764(C-Cl str.); ${ }^{1} \mathrm{H}$ NMR (400 MHz, DMSO, $\delta$ ppm): 10.79 (d, NH of tryptophan ring, $1 \mathrm{H}), 8.78 \& 8.32(\mathrm{~m}, \mathrm{NH}, 2 \mathrm{H}$, amide), $8.01(\mathrm{~s}, \mathrm{Ar}-\mathrm{H}, 1 \mathrm{H}$, aromatic), 8.11-
7.69 (m, Ar-H, 4H, quinazoline), 7.51-7.42 (m, Ar-H, 4H, chlorobenzene), 7.25-7.09 (m, Ar-H, 2H), $5.82(\mathrm{~d}, \mathrm{CH}, 1 \mathrm{H}), 4.94(\mathrm{~m}, \mathrm{OH}, 1 \mathrm{H}), 4.26\left(\mathrm{t}, \mathrm{CH}_{2}\right.$, $2 \mathrm{H}), 3.71\left(\mathrm{~s}, \mathrm{CH}_{3}, 3 \mathrm{H}\right)$.

\section{In vitro antimicrobial activity}

Newly synthesized amino acid and peptide derivatives of quinazolinone $8 \mathrm{a}-\mathrm{b}$ and $11 \mathrm{a}-\mathrm{b}$ were screened for their antimicrobial potential by disc diffusion method against Gram-positive bacteria such as Streptococcus pyogenes, Staphylococcus aureus, and Gram-negative bacteria like Pseudomonas aeruginosa, Escherichia coli at a concentration of $100 \mathrm{\mu gml}^{-1}$. The test compounds were dissolved in an appropriate solvent and were incubated at $37^{\circ} \mathrm{C}$ for $24 \mathrm{hrs}$. After incubation, the zone of inhibition was measured in $\mathrm{mm}$ and was compared with standard drug ciprofloxacin $(10 \mu \mathrm{g} / \mathrm{ml})$. The results have been summarized in Table 2.

\section{RESULTS AND DISCUSSION}

\section{Chemistry}

Four novel amino acid and peptide derivatives of qinazoline-4(3H)one were synthesized by condensation of 5-(2-(2-chlorophenyl)-4oxoquinazolin-3(4H)-yl)-2-hydroxy benzoic acid with different amino acid/dipeptide methyl ester in the presence of NMM and DCC as a coupling agent. The temperature of reaction mixture was maintained at $0^{\circ} \mathrm{C}$ for $36 \mathrm{hrs}$ with continuous stirring. The yield of all synthesized compounds was in the range of $65-76 \%$. The purification of all compounds was carried out by recrystallization in a suitable solvent and structures of synthesized compounds were confirmed by spectral techniques including IR and ${ }^{1} \mathrm{H}$ NMR.

The formation of qinazoline-4(3H)-one scaffold was confirmed by appearance of characteristic IR absorption bands at 3356-3285 $\mathrm{cm}^{-1}$ $(\mathrm{O}-\mathrm{H}), 1665 \mathrm{~cm}^{-1}$ ( $\mathrm{C}=\mathrm{O}$ amide), and $1725 \mathrm{~cm}^{-1}$ ( $\mathrm{C}=0$ ester). In ${ }^{1} \mathrm{H}$ NMR, peaks at $\delta 12.1 \mathrm{ppm}$ indicated carboxyl proton, 8.13-7.64 ppm due aromatic protons of quinazolinone ring, 7.39-7.36 ppm corresponding to benzene ring protons, and at $3.8 \mathrm{ppm}$ due to hydroxyl proton. The coupling of quinazolinone nucleus with amino acids/peptides was 
confirmed by disappearance of $\mathrm{NH}_{2}$ and $\mathrm{COOH}$ signals and appearance of CONH signals in both IR and NMR spectra.

\section{Biological activity}

All the synthesized compounds 8a-b and 11a-b were evaluated for their in vitro antimicrobial potential against both Gram-positive and Gram-negative bacteria such as $S$. pyogenes, $S$. aureus P. aeruginosa, and $E$. coli. Both amino acid and peptide derivatives exhibited excellent to moderate antimicrobial activity as compared to the test drug ciprofloxacin. The compound 11b was found to be most potent against all test microorganisms. It is expected due to the presence of indole moiety. The ester functionality present in designed peptide chain might undergo hydrolysis to give carboxylic group which form zwitterion by proton transfer to basic indole moiety, thereby exhibited broad spectrum antibacterial activity similar to most of quinolones. The moderate biological activity of other synthesized derivatives was expected to be due to the presence of nitrogen containing six membered fused heterocyclic ring of quinazolinone. The introduction of amino acid/ peptide chain substitutions at 3-position of quinazolinone ring gives polar character to the molecule which helps in permeation through bacterial cell membrane via densely charged porin channels [40]. The compound $11 \mathrm{a}$ has shown maximum activity against $P$. aeruginosa while 8a against $S$. aureus bacteria. All the synthesized compounds were found to be least active against $S$. pyogenes.

\section{CONCLUSION}

In summary, a series of amino acid and peptide derivatives of 5-(2-(2-chlorophenyl)-4-oxoquinazolin-3(4H)-yl)-2-hydroxy benzoic acid were synthesized by coupling reaction and screened for their antimicrobial potential against $S$. pyogenes, S. aureus, P. aeruginosa, and E. coli. All the derivatives have shown promising antimicrobial activity; compound $11 \mathrm{~b}$ has shown maximum antimicrobial activity against both Gram-positive and Gram-negative bacteria whereas compound 11a was more active against Gram-negative bacteria and 8a against Gram-positive bacteria.

\section{ACKNOWLEDGMENT}

The authors express sincere gratitude to the management of Lovely Professional University, for providing essential requirements for carrying out this research work.

\section{REFERENCES}

1. Akinyele TA, Igbinosa EO, Akinpelu DA, Okoh AI. In vitro assessment of the synergism between extracts of Cocos nucifera husk and some standard antibiotics. Asian Pac J Trop Biomed 2017;7(4):306-13.

2. Fernandes P, Martens E. Antibiotics in late clinical development. Biochem Pharmacol 2017;133(1):152-63.

3. Rather IA, Kim BC, Bajpai VK, Park YH. Self-medication and antibiotic resistance: Crisis, current challenges, and prevention. Saudi J Biol Sci 2017. DOI: 10.1016/j.sjbs.2017.01.004.

4. Dafale NA, Semwal UP, Rajput RK, Singh GN. Selection of appropriate analytical tools to determine the potency and bioactivity of antibiotics and antibiotic resistance. J Pharm Anal 2016;6:207-13.

5. Fajardo A, Linares JF, Martínez JL. Towards an ecological approach to antibiotics and antibiotic resistance genes. Clin Microbiol Infect 2009;15 Suppl 1:14-6.

6. Bengtsson-Palme J, Larsson DG. Concentrations of antibiotics predicted to select for resistant bacteria: Proposed limits for environmental regulation. Environ Int 2016;86:140-9.

7. Abbas SE, Barsoum FF, Georgey HH, Mohammed ER. Synthesis and antitumor activity of certain 2, 3, 6-trisubstituted quinazolin-4 (3H)-one derivatives. Bull Fac Pharm 2013;51:273-82.

8. Banu BH, Bharathi K, Prasad KV. Synthesis, characterization and evaluation of in vitro antioxidant and anti-inflammatory activity of 2-(4-oxo-2-phenylquinazolin-3 (4H)-yl) substituted acetic acids. IOSR J Pharm 2012;2:97-104.

9. Faraj FL, Zahedifard M, Paydar M, Looi CY, Majid NA, Ali HM, et al. Synthesis, characterization, and anticancer activity of new quinazoline derivatives against MCF-7 cells. Sci World J 2014;14:1-15.

10. Arora R, Kapoor A, Gill NS, Rana AC. Quinazolinone: An overview.
Int Res J Pharm 2011;2(12):21-8

11. Saad HA, Osman NA, Moustafa AH. Synthesis and analgesic activity of some new pyrazoles and triazoles bearing a 6, 8-dibromo-2methylquinazoline moiety. Molecules 2011;16(12):10187-201.

12. Priya MG, Girija K, Ravichandran N. In vitro study of anti-inflammatory and antioxidant activity of 4-(3H)-quinazolinone derivatives. Rasayan J Chem 2011;4:418-24.

13. Rakesh KP, Manukumar HM, Gowda DC. Schiff's bases of quinazolinone derivatives: Synthesis and SAR studies of a novel series of potential anti-inflammatory and antioxidants. Bioorg Med Chem Lett 2015;25(5):1072-7.

14. Patel DR, Patel KC. Synthesis, characterization and in vitro antimicrobial screening of some new MCT reactive dyes bearing nitro quinazolinone moiety. J Saudi Chem Soc 2015;19(4):347-59.

15. Khodarahmi G, Jafari E, Hakimelahi G, Abedi D, Khajouei MR, Hassanzadeh F. Synthesis of some new quinazolinone derivatives and evaluation of their antimicrobial activities. Iran J Pharm Res 2012;11:789-97.

16. Wang D, Gao F. Quinazoline derivatives: Synthesis and bioactivities. Chem Cent J 2013;7:1-15

17. Kumar D, Mariappan G, Husain A, Monga J, Kumar S. Design, synthesis and cytotoxic evaluation of novel imidazolone fused quinazolinone derivatives. Arab J Chem 2017;10(3):344-50.

18. Keche AP, Kamble VM. Synthesis and anti-inflammatory and antimicrobial activities of some novel 2-methylquinazolin-4 (3H)-one derivatives bearing urea, thiourea and sulphonamide functionalities. Arab J Chem 2014. DOI: 10.1016/j.arabjc.2014.10.025.

19. Zayed MF, Hassan MH. Synthesis and biological evaluation studies of novel quinazolinone derivatives as antibacterial and anti-inflammatory agents. Saudi Pharm J 2014;22(2):157-62.

20. Devi K, Kachroo M. Synthesis and anti-tubercular activity of some new 2, 3-disubstituted quinazolinones. Pharm Chem 2014;6(5):353-9.

21. Refaie FM, Esmat AY, Gawad SM, Ibrahim AM, Mohamed MA. The anti-hyperlipidemic activities of $4(3 \mathrm{H})$ quinazolinone and two halogenated derivatives in rats. Lipids Health Dis 2005;4:1-11.

22. Gobinath M, Subramanian N, Alagarsamy V. Design, synthesis and H1-antihistaminic activity of novel 1-substituted-4-(3chlorophenyl)-[1,2,4] triazo [4,3-a] quinazolin-5(4H)-ones. J Saudi Chem Soc 2015;19:282-6.

23. Gulick RM. New antiretroviral drugs. Clin Microbiol Infect 2003;9(3):186-93

24. Laddha SS, Bhatnagar SP. Novel fused quinazolinones: Further studies on the anticonvulsant activity of 1,2,9,11-tetrasubstituted$7 \mathrm{H}$-thieno $[2,3: 4,5]$ pyrimido $[6,1 \mathrm{~b}]$-quinazolin-7-one and 1,3,10,12-tetrasubstituted-8H-pyrido $\quad[2,3: 4,5]$ pyrimido $[6,1 \mathrm{~b}]$ quinazolin-8-one. Future Med Chem 2010;2:565-73.

25. Kumar S, Kaur H, Kumar A. Synthesis of new azetidinonyl/ thiazolidinonyl quinazolinone derivatives as anti-parkinsonian agents. Arab J Chem 2012;5(4):475-84.

26. Chaudhary S, Singh RK, Gupta RK. Synthesis and biological activity of peptide derivatives of 2-hydroxy-5-(6-iodo-2-methyl4-oxoquinazolin-3 (4H)-yl) benzoic acid. Asian J Pharm Clin Res 2012;5:196-200.

27. Himaja M, Gupta N, Munirajeskhar D, Asif K, Sikarwar MS. Synthesis and biological evaluation of some N-methylated derivatives of thiazolylamino acids and peptides. J Pharm Sci Innov 2012;1:33-6.

28. Guzmán F, Barberis S, Illanes A. Peptide synthesis: Chemical or enzymatic. Electron J Biotechnol 2007;10(2):279-314.

29. Pasupuleti M, Schmidtchen A, Malmsten M. Antimicrobial peptides: Key components of the innate immune system. Crit Rev Biotechnol 2012;32(2):143-71.

30. Ozaki KI, Yamada Y, Oine T, Ishizuka T, Iwasawa Y. Studies on $4(1 \mathrm{H})-$ quinazolinones. Synthesis and anti-inflammatory activity of $4(1 \mathrm{H})-$ quinazolinone derivatives. J Med Chem 1985;28(5):568-76.

31. Patel NB, Barat GG. In vitro microbial studies of new pyrazolyl quinazolin-4 (3H) ones. J Saudi Chem Soc 2010;14(2):157-64.

32. Balalaie S, Saeedi S, Ramezanpour S. Synthesis of pseudo-peptides containing a quinazolinone skeleton via Ugi four-component reaction. Helv Chim Acta 2016;99:138-42.

33. Dahiya R, Mourya R. Synthetic studies on novel nitroquinazolinone analogs with antimicrobial potential. Bull Pharm Res 2013;3(2):51-7

34. Babu VV. Ultrasound accelerated synthesis of proteinogenic and $\alpha$, a-dialkylamino acid ester salts. Indian J Chem 2006;45B:1942-4.

35. Kaur R, Kaur K, Gupta L, Dhir N, Gaganpreet. Synthesis and biological evaluation of gallic acid peptide derivatives. Int J Pharm Sci Rev Res 
2014;28(1):16-21.

36. Dahiya R, Gautam H. Total synthesis and antimicrobial activity of a natural cycloheptapeptide of marine origin. Mar Drugs 2010;8(8):2384-94.

37. Sribalan R, Padmini V, Lavanya A, Ponnuvel K. Evaluation of antimicrobial activity of glycinate and carbonate derivatives of cholesterol: Synthesis and characterization. Saudi Pharm J 2016;24(6):658-68.

38. Routier S, Saugé L, Ayerbe N, Coudert G, Mérour JY. A mild and selective method for N-Boc deprotection. Tetrahedron Lett 2002;43:589-91.

39. Evans V, Mahon MF, Webster RL. A mild, copper-catalysed amide deprotection strategy: Use of tert-butyl as a protecting group. Tetrahedron 2014;70(41):7593-7.

40. Beale JM. Anti-infective agents. In: Block JH, Beale JM, Troy DB, editors. Wilson and Gisvold's Textbook of Organic Medicinal and Pharmaceutical Chemistry. $11^{\text {th }}$ ed. Philadelphia, PA: Lippincott Williams and Wilkins; 2015. p. 248-50. 